\title{
Association of genetic variants of vit $D$ binding protein (DBP/GC) and of the enzyme catalyzing its 25-hydroxylation (DCYP2R1) and serum vit $\mathrm{D}$ in postmenopausal women
}

\author{
Wen Xu, ${ }^{1}$ Jing Sun, ${ }^{1}$ Wenbo Wang, ${ }^{2}$ Xiran Wang, ${ }^{3}$ Yan Jiang, ${ }^{1}$ Wei Huang,,${ }^{4}$ Xin Zheng, ${ }^{5}$ \\ Qiuping Wang, ${ }^{6}$ Zhiwei Ning, ${ }^{7}$ Yu Pei, ${ }^{8}$ Min Nie, ${ }^{1}$ Mei Li, ${ }^{1}$ Ou Wang,,${ }^{1}$ Xiaoping Xing, ${ }^{1}$ \\ Wei Yu, ${ }^{9}$ Qiang Lin, ${ }^{9}$ Ling Xu, ${ }^{10}$ Weibo Xia ${ }^{1}$
}

\begin{abstract}
${ }^{1}$ Department of Endocrinology, Key Laboratory of Endocrinology, Ministry of Health, Peking Union Medical College Hospital, Chinese Academy of Medical Science; ${ }^{2}$ Department of Endocrinology, Peking University Shougang Hospital; ${ }^{3}$ Department of Cadre Unit, General Hospital of the second Artillery Force; ${ }^{4}$ Department of Endocrinology, Beiding Haidian Hospital; ${ }^{5}$ Department of Endocrinology, China Rehabilitation Research Center, ${ }^{6}$ Department of Endocrinology, Beijing Liangxiang Hospital; ${ }^{7}$ Department of Endocrinology, Beijing Chaoyang Hospital; ${ }^{8}$ Department of Geriatric Endocrinology, Chinese PLA General Hospital; ' ${ }^{9}$ Department of Radiology, Peking Union Medical College Hospital, Chinese Academy of Medical Science; ${ }^{10}$ Department of Gynaecology and Obstetrics, Peking Union Medical College Hospital, Chinese Academy of Medical Science; Beijing, China
\end{abstract}

\begin{abstract}
OBJECTIVE: To determine if GC (group-specific component globulin) and CYP2R1 genetic variants have an association with serum $25-\mathrm{OHD}_{3}$ levels, BMD or bone turnover markers in a population of Chinese postmenopausal women. DESIGN: We randomly selected 1494 postmenopausal women of the Han ethnic group from seven communities in Beijing. BMD was determined by dual energy $\mathrm{X}$-ray absorptiometry; serum bone turnover markers and 25-OHD were measured by the automated Roche electrochemiluminescence system; genotypes of GC and CYP2R1 were detected by the TaqMan allelic discrimination assay. Multiple statistic methods were used to test the associations of SNP genotypes and vitamin D levels. RESULTS: In our sample, $89.6 \%$ women had vitamin $D$ deficiency and another $9.8 \%$ had vitamin $D$ insufficiency. The variants of $\mathbf{r s 2 2 8 8 4 9}(\beta=0.105, P<0.001)$ in $\mathrm{GC}$ were significantly associated with serum $25-\mathrm{OHD}_{3}$ levels. Allele $\mathrm{G}$ of $\mathbf{r s 2 2 9 8 8 4 9}$ might be protective for serum $25-\mathrm{OHD}_{3}$ level. Among the haplotypes of rs222020-rs2298849, $\mathrm{CG}(\boldsymbol{\beta}=\mathbf{0 . 1 0 4}, P=0.001)$ corresponded to increasing serum 25-OHD 3 concentrations. CYP2R1 polymorphisms showed some significant association with serum $\beta$-CTX and P1NP levels. CONCLUSIONS: We found that GC variants had a significant association with serum $25-\mathrm{OHD}_{3}$ levels among postmenopausal women of the Han ethnic group in Beijing, while CYP2R1 variants were not found to be significant.
\end{abstract}

Key words: Association analysis, BMD, Bone turnover markers, CYP2R1, GC, SNP, Vitamin D

Address for correspondence:

Prof. Weibo Xia, Department of Endocrinology, Key Laboratory of Endocrinology, Ministry of Health, Peking Union Medical College Hospital, Chinese Academy of Medical Science, Beijing 100730 China, Tel.: 86-10-6915-5358, Fax: 86-10-6915-5358,

E-mail: xiaweibo@medmail.com.cn

Received 23-05-2013, Accepted 08-09-2013 


\section{INTRODUCTION}

Vitamin D deficiency is a very common health problem. It is reported that worldwide more than 1 billion children and adults are at risk of vitamin D deficiency. ${ }^{1}$ The situation is likely to be worse in China. According to a large sample (more than 3,000 individuals) cross-sectional study in older adults living in Beijing and Shanghai, the percentage of vitamin D deficiency and insufficiency were 69.2 and $24.4 \%$, respectively. ${ }^{2}$ Vitamin D deficiency may decrease peak bone mass in young people and increase the risk of osteoporosis, falls and fractures in the elderly and old. ${ }^{3}$ It also associated with increasing risk of several autoimmune, cardiovascular, infectious and metabolic diseases. ${ }^{1}$ Although the main cause of vitamin $\mathrm{D}$ deficiency is considered to be lack of sunlight exposure and dietary vitamin D intake, family and twin studies have shown that genetic factors might also affect vitamin levels. ${ }^{4-6}$

The genome-wide association study (GWAS) and other association studies have been carried out to determine the functional genes. Group-specific component globulin (GC), also called vitamin D binding protein (DBP), is a multifunctional protein which can bind to vitamin $\mathrm{D}$, maintain the vitamin $\mathrm{D}$ level in the human body and transport vitamin D to target tissues for processing and utilization. ${ }^{7}$ CYP2R1 is a member of cytochrome P450 families. It can catalyze the 25-hydroxylation of vitamin D with no sex and species differences and shows catalytic activity toward both vitamin $\mathrm{D}_{2}$ and $\mathrm{D}_{3} .{ }^{8}$ Recently, some studies have observed an association of GC and CYP2R1 variants with serum vitamin D concentration in European populations. ${ }^{9-11}$ However, it remains to be confirmed whether they have similar effects in Chinese populations. Based on the Peking Vertebral Fracture (PK-VF) study, which is a large-scale epidemiologic study with randomly selected postmenopausal women residing in communities in Beijing, we examined whether the association reported for GC (rs222020 and rs2298849) and CYP2R1 (rs12794714, rs10741657, rs1562902 and rs10766197) variants are also present in this sample. We also explored whether these variants have some relationship with bone mineral density (BMD) and bone turnover markers such as $\beta$-C-terminal telopeptide of type 1 collagen $(\beta-\mathrm{CTX})$ and procollagen type I N propeptide (P1NP).

\section{SUBJECTS AND METHODOLOGY}

\section{Participants}

Our participants were from the PK-VF cohort together with randomly selected postmenopausal women living in communities in seven districts of Beijing. Before sampling, we put up posts on the bulletin board of each community center to explain the nature of the proposed study. We then made telephone calls to invite the randomly selected participants to join the study. Age distribution of our selected participants was consistent with the composition of those communities. At the end the PK-VF study, 2070 postmenopausal women were recruited. Every participant completed a questionnaire concerning items such as age, years since menopause, fracture history and medication history. Height and weight of the participants were measured and recorded by assistant doctors or nurses. Menopause was defined as the absence of menstruation for at least one year. Exclusion criteria were: 1) chronic liver disease; 2) chronic renal disease; 3 ) significant gastrointestinal diseases such as inflammatory bowel disease (IBD) and chronic diarrhea; 4) metabolic or inherited bone disease; 5) corticosteroid, anticonvulsant or antituberculosis therapy for more than 6 months during the previous year. Finally, 1546 participants were included in our study. The study was approved by the Ethics Committee of the Peking Union Medical College Hospital (PUMCH). All participants signed informed consent forms before entering the study.

\section{Biochemistry}

We collected a fasting blood sample from all the participants. Serum concentrations of 25-hydroxyvitamin D3 (25-OHD $)$, $\beta$-CTX and P1NP were measured by a fully automated Roche electrochemiluminescence system (E170, Roche Diagnostics, Switzerland) at PUMCH. The detection limit of $\beta$-CTX, P1NP and $25-\mathrm{OHD}_{3}$ was $0.01 \mathrm{ng} / \mathrm{ml}, 5 \mathrm{ng} / \mathrm{ml}$ and $4 \mathrm{ng} / \mathrm{ml}$, respectively. The intraassay and interassay $\mathrm{CV}$ were $2 \%$ and $3.1 \%$ for $\beta$-CTX, $2.3 \%$ and $1.7 \%$ for P1NP and $5.7 \%$ and $6.1 \%$ for $25-\mathrm{OHD}_{3}$.

\section{BMD measurement}

Details of BMD measurement have been described previously..$^{12}$ Briefly, we measured the BMD of the lumbar spine (L2-4), femoral neck (FN) and total hip by dual energy X-ray absorptiometry (DXA). 


\section{Genotyping}

We searched for single nucleotide polymorphisms (SNP) information on the GC and CYP2R1 genes on the HapMap website (http://hapmap.ncbi.nlm. nih.gov/). SNPs were selected according to the following criteria: 1) high heterozygosity, which means minor allele frequency (MAF) higher than $20 \%$ in the Chinese population (CHB in HapMap); 2) classified as tag SNPs; 3 ) reported to be associated with serum $25-\mathrm{OHD}_{3}$ level in previous studies. ${ }^{9-11}$ Finally, we selected two SNPs of the GC gene (rs222020, rs2298849) and four SNPs of the CYP2R1 gene (rs12794714, rs10741657, rs1562902, rs10766197). Each participant was genotyped for all six SNPs by TaqMan allelic discrimination assay (Applied Biosystems, USA). The whole reacting volume was $6 \mu 1$, including $3 \mu \mathrm{l}$ (approximately $15 \mathrm{ng}$ ) sample DNA, 2.5 $\mu$ l TaqMan Universal PCR Master Mix, 0.0875 $\mu \mathrm{l}$

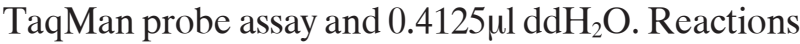
were performed on a Real-Time PCR system of ABI Prism 7900 (Applied Biosystems, USA) in a 384-well reaction plate under standard conditions. The genotyping success rate for six SNPs was higher than $98 \%$, and the concordance rate was about $94.4 \%$ based on $3 \%$ duplicated samples. Genotype frequencies of the six SNPs were similar to those in HapMap-CHB (http://hapmap.ncbi.nlm.nih.gov/) and they were all in Hardy-Weinberg equilibrium.

\section{Statistics}

The Hardy-Weinberg equilibrium (HWE) was tested using the goodness-of-fit chi-square test of the Haploview 4.2 program, which was also used to calculate normalized LD coefficient (D'), and the Pearson correlation coefficient $\left(\mathrm{r}^{2}\right)$ to measure link- age disequilibrium (LD) between pairwise SNPs. Haplotypes of each participant were determined by Phase 2.02. Serum $25-\mathrm{OHD}_{3}$ levels were squareroot transformed to approximate normality. The independent samples t-test was performed to test the difference in serum $25-\mathrm{OHD}_{3}$ levels between different seasons. To establish the potential effect of numeric covariates on serum $25-\mathrm{OHD}_{3}$ levels, a Pearson correlation was conducted as an exploratory analysis (SPSS, version 17.0). Age, BMI and blood collecting season (winter-spring defined as 1 , or summer defined as 2) were considered as potential covariates in a linear regression model. Association of the square-root-transformed serum $25-\mathrm{OHD}_{3}$ levels with age, season and all the SNPs and haplotypes were tested by general linear regression using backward elimination in SPSS, respectively. We also conducted the Pearson correlation or general linear regression to test correlation or association of the 6 SNPs with $\beta$-CTX, P1NP and BMD of L2-4, femoral neck and total hip. All analyses were carried out under an additive model. $\mathrm{P}<0.05$ was considered statistically significant. The statistical power was calculated by Quanto software (http://hydra.usc.edu/gxe/). Our study achieved a power of more than $80 \%$.

\section{RESULTS}

\section{Basic characteristics of participants}

After excluding participants who failed the biochemistry test or genotyping, 1494 participants remained. The major clinical profiles of these participants are listed in Table 1. Among them, 1338 women had vitamin D deficiency $\left(25-\mathrm{OHD}_{3}<20 \mathrm{ng} /\right.$ $\mathrm{ml}$ ) and another 146 women had vitamin D insuffi-

Table 1. Basic characteristics of participants

\begin{tabular}{lclc}
\hline Characteristics & Mean \pm SD & Characteristics & Mean \pm SD \\
\hline Age $($ years $)(\mathrm{n}=1494)$ & $64.0 \pm 9.2$ & Vitamin D deficiency $(\mathrm{n}, \%)$ & $1338,89.6 \%$ \\
Height $(\mathrm{cm})(\mathrm{n}=1494)$ & $155.1 \pm 5.6$ & Vitamin D insufficiency $(\mathrm{n}, \%)$ & $146,9.8 \%$ \\
Weight $(\mathrm{kg})(\mathrm{n}=1494)$ & $62.8 \pm 9.8$ & $\beta-C T X(\mathrm{ng} / \mathrm{ml})(\mathrm{n}=1494)$ & $0.440 \pm 0.212$ \\
Body mass index $\left(\mathrm{kg} / \mathrm{m}^{2}\right)(\mathrm{n}=1494)$ & $26.1 \pm 3.7$ & P1NP $(\mathrm{ng} / \mathrm{ml})(\mathrm{n}=1494)$ & $56.95 \pm 28.70$ \\
Serum 25-OHD $3(\mathrm{ng} / \mathrm{ml})(\mathrm{n}=1494)$ & $13.2 \pm 5.4$ & BMD of L2-4 $\left(\mathrm{g} / \mathrm{cm}^{2}\right)(\mathrm{n}=1452)$ & $0.966 \pm 0.198$ \\
Winter-Spring $($ Dec-May) $(\mathrm{n}=805)$ & $12.8 \pm 5.2$ & BMD of FN $\left(\mathrm{g} / \mathrm{cm}^{2}\right)(\mathrm{n}=1451)$ & $0.767 \pm 0.139$ \\
Summer $(J u n-A u g)(\mathrm{n}=689)$ & $13.7 \pm 5.6$ & BMD of TH $\left(\mathrm{g} / \mathrm{cm}^{2}\right)(\mathrm{n}=985)$ & $0.865 \pm 0.144$ \\
\hline
\end{tabular}

Vitamin D deficiency: 25-OHD $3<20 \mathrm{ng} / \mathrm{ml}$; vitamin D insufficiency: 20ng/ml $\leqq 25-\mathrm{OHD}_{3}<30 \mathrm{ng} / \mathrm{ml}$. 
ciency $\left(20 \mathrm{ng} / \mathrm{ml} \leqq 25-\mathrm{OHD}_{3}<30 \mathrm{ng} / \mathrm{ml}\right)$, accounting for $89.6 \%$ and $9.8 \%$ of the total sample, respectively.

Among the 1494 participants, 91 blood samples were collected in winter, while 714 and 689 blood samples were collected in spring and summer, respectively. Since the sample size of the winter group was small and there was no significantly statistical difference between the winter and spring groups after adjusting age and BMI, we divided the 1494 participants into two season groups: summer and winter-spring. The concentrations of serum $25-\mathrm{OHD}_{3}$ were significantly different between the summer group and the winter-spring group $(P=0.001)$.

\section{Serum 25-OHD3 levels in different seasons}

Because the sample size of the winter group was small, and there was no significantly statistical difference of serum $25-\mathrm{OHD}_{3}$ levels between the winter and spring groups $(P=0.671)$, we divided the 1494 participants into two season groups: summer and winter-spring. The serum $25-\mathrm{OHD}_{3}$ levels were significantly different between the summer and the winter-spring groups $(P=0.002)$, as shown in Table 2 .

\section{Association of GC and CYP2R1 genetic variants with serum $25-\mathrm{OHD}_{3}$ levels}

To determine the potential effect of numeric covariates on serum $25-\mathrm{OHD}_{3}$ levels, a Pearson correlation was conducted as an exploratory analysis (SPSS, version 17.0). Except for all the SNPs under the additive model, age, BMI and blood collecting
Table 2. Result of t-test of serum $25-\mathrm{OHD}_{3}$ levels in different seasons

\begin{tabular}{lcccc}
\hline $\begin{array}{l}\text { Blood collecting } \\
\text { season }\end{array}$ & $\mathbf{N}$ & $\begin{array}{c}\mathbf{2 5 - O H D} \\
(\mathbf{n g} / \mathbf{m l})\end{array}$ & $\begin{array}{c}\text { Sqrt of 25-OHD } \\
(\mathbf{n g} / \mathbf{m l})\end{array}$ & $\boldsymbol{P}$ \\
\hline Winter & 91 & $13.1 \pm 5.7$ & $3.54 \pm 0.80$ & 0.671 \\
Spring & 714 & $12.8 \pm 5.1$ & $3.50 \pm 0.72$ & \\
$\begin{array}{l}\text { Winter - Spring } \\
\text { (Dec-May) }\end{array}$ & 805 & $12.8 \pm 5.2$ & $3.51 \pm 0.73$ & \\
$\begin{array}{l}\text { Summer } \\
\text { (Jun-Aug) }\end{array}$ & 689 & $13.7 \pm 5.6$ & $3.62 \pm 0.77$ & $\mathbf{0 . 0 0 2}$ \\
\hline Sqrt of 25-OHD : square-root-transformed serum 25-OHD level.
\end{tabular}

season (winter-spring defined as 1, or summer defined as 2) were considered as potential covariates in a linear regression model. In our study, SNPs rs222020 $(\mathrm{r}=0.080, P=0.001)$ and $\mathrm{rs} 2298849(\mathrm{r}=0.095, P<0.001)$ of the GC gene, age $(\mathrm{r}=-0.074, P=0.002)$ and blood collecting season $(\mathrm{r}=0.079, P=0.001)$ were found to have significant correlation with serum $25-\mathrm{OHD}_{3}$ levels. Following this, the association of the squareroot-transformed serum $25-\mathrm{OHD}_{3}$ levels with age, season and all the SNPs were tested by general linear regression using backward elimination in SPSS. The main results of the SNPs genotyping of the GC and the CYP2R1 genes and their association analyses for serum $25-\mathrm{OHD}_{3}$ levels are shown in Table 3. SNP rs2298849 $(\beta=0.105, P<0.001)$ of the GC gene was found to have a significant association with serum $25-\mathrm{OHD}_{3}$ levels. We did not find any significant association between CYP2R1 genetic variants and serum $25-\mathrm{OHD}_{3}$ levels.

Table 3. Results of single SNPs association analyses for serum $25-\mathrm{OHD}_{3}$ levels

\begin{tabular}{|c|c|c|c|c|c|c|c|c|}
\hline Covariates & & & & & $\mathbf{r}$ & $P 1$ & $\beta(\mathrm{SE})$ & $P 2$ \\
\hline Genes & SNP & Allele & MAF & HWE & & & & \\
\hline $\mathrm{GC}$ & rs 222020 & $\mathrm{~T} / \mathrm{C}$ & 0.338 & 0.86 & 0.080 & 0.001 & & \\
\hline $\mathrm{GC}$ & rs 2298849 & $\mathrm{~A} / \mathrm{G}$ & 0.324 & 0.55 & 0.095 & $<0.001$ & $0.105(0.029)$ & $<0.001$ \\
\hline CYP2R1 & rs12794714 & $\mathrm{G} / \mathrm{A}$ & 0.373 & 0.87 & -0.010 & 0.353 & & \\
\hline CYP2R1 & rs 10741657 & $\mathrm{G} / \mathrm{A}$ & 0.396 & 0.96 & -0.007 & 0.387 & & \\
\hline CYP2R1 & rs1562902 & $\mathrm{T} / \mathrm{C}$ & 0.435 & 0.83 & -0.016 & 0.272 & & \\
\hline CYP2R1 & rs10766197 & $\mathrm{G} / \mathrm{A}$ & 0.371 & 0.51 & -0.014 & 0.296 & & \\
\hline Age & & & & & -0.074 & 0.002 & $-0.006(0.002)$ & 0.003 \\
\hline Season & & & & & 0.079 & 0.001 & $0.127(0.039)$ & 0.001 \\
\hline
\end{tabular}

Serum 25-OHD 3 levels were square-root transformed to approximate normality. Bold numbers represent significant $P$ values. Allele: major allele/minor allele; MAF: minor allele frequency; HWE: $P$ values for Hardy-Weinberg Equilibrium test; r: Pearson correlation coefficient; $P 1: P$ value of Pearson correlation; $\beta$ : regression coefficient; SE: standard error; $P 2: P$ value of general linear regression. 
The LD plots of the GC and CYP2R1 genes are depicted in Figure 1. The results of the haplotype association analyses are displayed in Table 4 . The two SNPs of the GC gene were in relatively strong (a) $\mathrm{GC}$
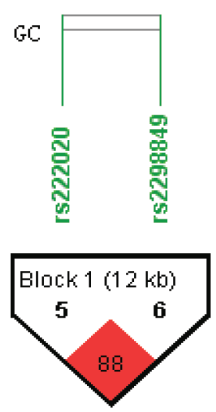

(b) CYP2R1
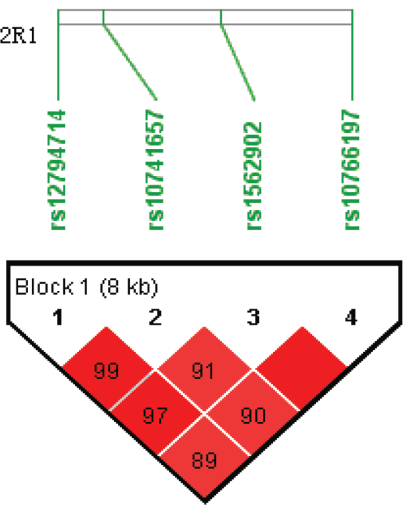

Figure 1. LD plots with D' values of the GC (a) and CYP2R1 (b) genes in our 1494 participants. The Figure was generated by Haploview. Block definition followed the rules of solid spine of LD. D' values multiplied by 100 are shown as a number in the diamonds.

Table 4. Results of haplotypes association analyses for serum 25$\mathrm{OHD}_{3}$ levels

\begin{tabular}{|c|c|c|c|c|c|}
\hline Covariates & & $\mathbf{r}$ & $P 1$ & $\beta(\mathrm{SE})$ & $P 2$ \\
\hline \multicolumn{6}{|c|}{ GC: rs222020-rs2298849 } \\
\hline Haplotype & Frequency & & & & \\
\hline TA & 0.638 & -0.082 & 0.001 & & \\
\hline CG & 0.299 & 0.094 & $<0.001$ & $\begin{array}{c}0.104 \\
(0.030)\end{array}$ & 0.001 \\
\hline CA & 0.038 & -0.024 & 0.173 & & \\
\hline $\mathrm{TG}$ & 0.024 & 0.012 & 0.322 & & \\
\hline
\end{tabular}

CYP2R1: rs12794714-rs10741657-rs1562902-rs10766197

Haplotype Frequency

\begin{tabular}{llcccc} 
GACG & 0.375 & -0.004 & 0.444 & & \\
AGTA & 0.347 & -0.019 & 0.233 & & \\
GGTG & 0.165 & 0.030 & 0.124 & & \\
GGCG & 0.057 & -0.017 & 0.250 & & \\
& & -0.074 & $\mathbf{0 . 0 0 2}$ & -0.006 & $\mathbf{0 . 0 0 3}$ \\
Age & & & & $(0.002)$ & \\
Season & & 0.079 & $\mathbf{0 . 0 0 1}$ & 0.125 & $\mathbf{0 . 0 0 1}$ \\
& & & & $(0.039)$ & \\
\hline
\end{tabular}

Serum $25-\mathrm{OHD}_{3}$ levels were square-root transformed to approximate normality. Bold numbers represent significant $P$ values. r: Pearson correlation coefficient; $P 1: P$ value of Pearson correlation; $\beta$ : regression coefficient; SE: standard error; $P 2: P$ value of general linear regression.
LD (D'>0.8) with each other, as were also the four SNPs of the CYP2R1 gene. $P$ values were calculated using general linear regression (SPSS, version 17.0) under an additive model for those haplotypes with more than $1 \%$ frequency. Among the haplotypes of rs222020-rs2298849, CG ( $\beta=0.104, P=0.001)$ corresponded to increasing serum $25-\mathrm{OHD}_{3}$ concentrations. We did not find any significant association between haplotypes of the CYP2R1 gene and serum $25-\mathrm{OHD}_{3}$ levels.

\section{Association of GC and CYP2R1 genetic variants with bone turnover markers and BMD}

Basic information concerning P1NP, $\beta$-CTX and BMD of the lumbar spine (L2-4), femoral neck (FN) and total hip $(\mathrm{TH})$ is shown in Table 1. Some participants failed to attend the BMD test, therefore the available data of BMD was limited, especially as regards $\mathrm{BMD}$ of $\mathrm{TH}$.

To determine the potential effect of numeric covariates on bone turnover markers and BMD, a Pearson correlation was conducted (SPSS, version 17.0) as an exploratory analysis. Age and BMI were considered as potential covariates.

As shown in Table 5, in our research SNP $\mathrm{rs} 12794714(\mathrm{r}=-0.053, P=0.021)$ and $\mathrm{BMI}(\mathrm{r}=-0.052$, $P=0.022$ ) were found to have significant correlation with $\beta$-CTX levels; SNPs rs12794714 ( $\mathrm{r}=-0.064$, $P=0.007)$ and $\mathrm{rs} 10741657(\mathrm{r}=0.046, P=0.036)$ had significant correlation with P1NP levels. In the final linear regression model, SNPs rs12794714 $(\beta=-0.056$, $P=0.002)$ and $\operatorname{rs} 10766197(\beta=0.044, P=0.012)$ had statistically significant association with $\beta$-CTX levels; SNP rs12794714 ( $\beta=-0.017, P=0.014)$ had statistically significant association with P1NP levels. Although we found some statistically significant results, as the magnitude of association was rather small, we did not do further haplotype association analysis.

As shown in Table 6, in our research, age $(\mathrm{r}=-0.299$, $P<0.001)$ and BMI $(\mathrm{r}=0.183, P<0.001)$ had significant correlation with BMD of L2-4; age ( $\mathrm{r}=-0.521$, $P<0.001)$ and BMI $(\mathrm{r}=0.210, P<0.001)$ had significant correlation with BMD of FN; age $(\mathrm{r}=-0.535, P<0.001)$ and BMI $(\mathrm{r}=0.272, P<0.001)$ had significant correlation with BMD of TH. None of the 6 SNPs were found to have significant correlation with BMD of 
Table 5. Results of single SNPs association analyses for $\beta$-CTX and P1NP levels

\begin{tabular}{|c|c|c|c|c|c|c|c|c|c|}
\hline \multirow{2}{*}{$\begin{array}{l}\text { Dependent } \\
\text { Covariates }\end{array}$} & & \multicolumn{4}{|c|}{$\beta$-CTX } & \multicolumn{4}{|c|}{ P1NP } \\
\hline & & $\mathbf{r}$ & $P 1$ & $\beta(\mathrm{SE})$ & $P 2$ & $\mathbf{r}$ & $P 1$ & $\beta(\mathrm{SE})$ & $P 2$ \\
\hline Gene & SNP & & & & & & & & \\
\hline GC & rs222020 & 0.010 & 0.349 & & & 0.008 & 0.379 & & \\
\hline GC & rs2298849 & -0.004 & 0.436 & & & -0.003 & 0.457 & & \\
\hline CYP2R1 & rs12794714 & -0.053 & 0.021 & $-0.056(0.018)$ & 0.002 & -0.064 & 0.007 & $-0.017(0.007)$ & 0.014 \\
\hline CYP2R1 & rs10741657 & 0.036 & 0.084 & & & 0.046 & 0.036 & & \\
\hline CYP2R1 & rs1562902 & 0.025 & 0.163 & & & 0.036 & 0.085 & & \\
\hline CYP2R1 & rs10766197 & -0.018 & 0.246 & $0.044(0.018)$ & 0.012 & -0.043 & 0.050 & & \\
\hline Age & & 0.029 & 0.128 & & & 0.030 & 0.121 & & \\
\hline BMI & & -0.052 & 0.022 & $-0.003(0.001)$ & 0.051 & 0.010 & 0.351 & & \\
\hline
\end{tabular}

P1NP levels were log-transformed to approximate normality. Bold numbers represent significant $P$ values.

r: Pearson correlation coefficient; $P 1: P$ value of Pearson correlation; $\beta$ : regression coefficient; SE: standard error; $P 2: P$ value of general linear regression.

Table 6. Results of single SNPs correlation analyses for BMD of L2-4, FN, and TH

\begin{tabular}{|c|c|c|c|c|c|c|c|}
\hline \multirow{2}{*}{$\begin{array}{l}\text { Dependent } \\
\text { Covariates }\end{array}$} & & \multicolumn{2}{|c|}{ L2-4 BMD (N=1452) } & \multicolumn{2}{|c|}{ FN BMD $(\mathrm{N}=1451)$} & \multicolumn{2}{|c|}{ TH BMD $(\mathrm{N}=985)$} \\
\hline & & $\mathbf{r}$ & $P$ & $\mathbf{r}$ & $P$ & $\mathbf{r}$ & $P$ \\
\hline Gene & SNP & & & & & & \\
\hline GC & rs222020 & -0.019 & 0.231 & 0.014 & 0.291 & -0.006 & 0.430 \\
\hline GC & rs2298849 & -0.019 & 0.237 & 0.009 & 0.361 & -0.013 & 0.347 \\
\hline CYP2R1 & rs12794714 & 0.005 & 0.431 & 0.017 & 0.261 & 0.031 & 0.163 \\
\hline CYP2R1 & rs10741657 & 0.003 & 0.452 & -0.014 & 0.301 & 0.001 & 0.491 \\
\hline CYP2R1 & rs1562902 & 0.001 & 0.492 & -0.025 & 0.173 & -0.033 & 0.149 \\
\hline CYP2R1 & rs10766197 & -0.002 & 0.463 & 0.011 & 0.344 & 0.038 & 0.118 \\
\hline Age & & -0.299 & $<0.001$ & -0.521 & $<0.001$ & -0.535 & $<0.001$ \\
\hline BMI & & 0.183 & $<0.001$ & 0.210 & $<0.001$ & 0.272 & $<0.001$ \\
\hline
\end{tabular}

Bold numbers represent significant $P$ values. r: Pearson correlation coefficient; $P$ : $P$ value of Pearson correlation.

L2-4, FN, or TH $(P>0.05)$. We thus did not proceed to further linear regression to test the association between SNPs or haplotypes and BMD.

\section{DISCUSSION}

In our study, $89.6 \%$ of the 1494 women had vitamin D deficiency, which suggests that vitamin D deficiency might be very common in postmenopausal women in Beijing. percentage is higher than the $69.2 \%$ reported in a previous study by $\mathrm{Lu}$ et al. in a Chinese population. ${ }^{2}$ The observed difference might be due to age, latitude, sex and blood collecting season. The average age of our sample was about 64 years old, which is higher than the 59 years old of their study. Participants of our study were residents of Beijing, while theirs lived in Beijing and Shanghai and, as is known, Beijing is at higher latitude than Shanghai. Our participants were all women, while they included both men and women. They collected blood samples mainly from April to June, which is characterized by relatively abundant sunlight exposure, while we collected blood samples over a large scale of time with less sunlight, from December to October.

We found that the variants of $\operatorname{rs} 2298849(\beta=0.105$, $P<0.001)$ in the GC gene were significantly associated with serum $25-\mathrm{OHD}_{3}$ levels. Allele G of rs 2298849 
might be protective for serum $25-\mathrm{OHD}_{3}$ levels. The variants of $\mathrm{rs} 222020(\mathrm{r}=0.080, P=0.001)$ in the $\mathrm{GC}$ gene were correlated with serum $25-\mathrm{OHD}_{3}$ levels, although they were not significantly associated with serum $25-\mathrm{OHD}_{3}$ levels in the final linear regression model. This finding is consistent with the study by $\mathrm{Bu}$ et al. ${ }^{11}$ Previous studies on the GC gene focused more on functional SNP rs4588 located in the exon. Its A/ Lyn allele corresponds to the GC2 protein, which was found to be significantly associated with decreased serum $25-\mathrm{OHD}_{3}$ level ${ }^{7,14,15} \mathrm{Lu}$ et al. observed that the variants of rs 4588 were associated with plasma $25-\mathrm{OHD}_{3}$ levels in a Chinese population, ${ }^{16}$ while Ahn et al. and Wang et al. found that rs 2282679 variants were associated with serum $25-\mathrm{OHD}_{3}$ levels in white Europeans. ${ }^{910}$ In the HapMap Data, rs2282679 and rs4588 are in complete LD $\left(\mathrm{r}^{2}=1\right)$ in CEU and CHB populations, while these two SNPs are both in very weak LD with rs222020 and rs2298849 $\left(\mathrm{r}^{2}\right.$ ranging from 0.08 to 0.15 ). As rs222020 and rs2298849 are both located in the intron, there might be some new functional site which is independent of rs4588 and can influence serum $25-\mathrm{OHD}_{3}$ levels. Fine genotyping in the area near rs222020 and rs2298849 might need to be done to discover new functional sites.

We detected no significant association between CYP2R1 polymorphisms and serum $25-\mathrm{OHD}_{3}$ levels in Chinese postmenopausal women. This finding is consistent with the previous study in a Chinese population by Lu et al. ${ }^{16}$ However, CYP2R1 genetic variants were found to be associated with vitamin $\mathrm{D}$ level in many studies in white Europeans. ${ }^{9-11}$ Since we had $>99 \%$ power to find the beta values reported by $\mathrm{Bu}$ et al. in white Europeans, ${ }^{11}$ the discrepancy with previous studies may result from LD pattern variation of the causal SNP and the four SNPs detected in our study between Chinese Hans and white Europeans. Previous studies have hypothesized that genetic factors may play a much more important role in influencing vitamin D level in men than in women. ${ }^{4}$ As we had only female participants, our results may not represent the status of men. Another possibility is that other vitamin D 25-hydroxylation enzymes play a more important role in Chinese Hans than in white Europeans, thus CYP2R1 polymorphisms would hypothetically not influence serum $25-\mathrm{OHD}_{3}$ level in Chinese Hans.
In our study, we found CYP2R1 polymorphisms to be significantly associated with two bone turnover markers: $\beta$-CTX and P1NP. As we discovered no significant association between CYP2R1 polymorphisms and serum $25-\mathrm{OHD}_{3}$ levels in our study, the CYP2R1 gene might influence these two bone turnover markers via some as yet unknown mechanism. Since we did not locate any previous studies on the association between CYP2R1 genetic polymorphisms and the two bone turnover markers, this result may need to be further corroborated considering the small magnitude of the association.

\section{CONCLUSIONS}

In conclusion, we found that $\mathrm{GC}$ genetic variants had a significant association with serum $25-\mathrm{OHD}_{3}$ level among postmenopausal women of the Han ethnic group in Beijing, while CYP2R1 genetic variants were not observed as being significant. Association of CYP2R1 genetic variants with $\beta$-CTX and P1NP needs to be further substantiated.

\section{GRANTS SUPPORTS}

This research was supported by the National Natural Science Foundation of China (No.81070687 and 81170805), National Science and Technology Pillar Program (2006BAI02B03), National Science and Technology Major Projects for "Major New Drugs Innovation and Development" (Grant2008ZX09312-016), Beijing Natural Science Foundation (No. 7121012) and Scientific Research Foundation of Beijing Medical Development (No. 2007-3029).

\section{REFERENCES}

1. Holick MF, 2011 Vitamin D deficiency in 2010: health benefits of vitamin D and sunlight: a D-bate. Nat Rev Endocrinol 7: 73-75.

2. Lu L, Yu Z, Pan A, et al, 2009 Plasma 25-hydroxyvitamin $\mathrm{D}$ concentration and metabolic syndrome among middle-aged and elderly Chinese individuals. Diabetes Care 32: 1278-1283.

3. Holick MF, 2011 Vitamin D: a d-lightful solution for health. J Investig Med 59: 872-880.

4. Arguelles LM, Langman CB, Ariza AJ, et al, 2009 Heritability and environmental factors affecting vitamin D status in rural Chinese adolescent twins. J Clin Endocrinol Metab 94: 3273-3281. 
5. Shea MK, Benjamin EJ, Dupuis J, et al, 2009 Genetic and non-genetic correlates of vitamins $\mathrm{K}$ and D. Eur J Clin Nutr 63: 458-464.

6. Snellman G, Melhus H, Gedeborg R, et al, 2009 Seasonal genetic influence on serum 25-hydroxyvitamin D levels: a twin study. PLoS One 4: e7747.

7. Fang Y, van Meurs JB, Arp P, et al, 2009 Vitamin D binding protein genotype and osteoporosis. Calcif Tissue Int 85: 85-93.

8. Strushkevich N, Usanov SA, Plotnikov AN, Jones G, Park HW, 2008 Structural analysis of CYP2R1 in complex with vitamin D3. J Mol Biol 380: 95-106.

9. Ahn J, Yu K, Stolzenberg-Solomon R, et al, 2010 Genome-wide association study of circulating vitamin D levels. Hum Mol Genet 19: 2739-2745.

10. Wang TJ, Zhang F, Richards JB, et al, 2010 Common genetic determinants of vitamin D insufficiency: a genome-wide association study. Lancet 376: 180-188.

11. Bu FX, Armas L, Lappe J, et al, 2010 Comprehensive association analysis of nine candidate genes with serum 25-hydroxy vitamin D levels among healthy Caucasian subjects. Hum Genet 128: 549-56.
12. Zhao J, Xia W, Nie M, et al, 2012 A haplotype of MATN3 is associated with vertebral fracture in Chinese postmenopausal women: Peking Vertebral Fracture (PK-VF) study. Bone 50: 917-924.

13. Purcell S, Neale B, Todd-Brown K, et al, 2007 PLINK: a tool set for whole-genome association and populationbased linkage analyses. Am J Hum Genet 81: 559-575.

14. Abbas S, Linseisen J, Slanger T, et al, 2008 The Gc2 allele of the vitamin $\mathrm{D}$ binding protein is associated with a decreased postmenopausal breast cancer risk, independent of the vitamin D status. Cancer Epidemiol Biomarkers Prev 17: 1339-1343.

15. Lauridsen AL, Vestergaard P, Hermann AP, et al, 2005 Plasma concentrations of 25-hydroxy-vitamin D and 1,25-dihydroxy-vitamin D are related to the phenotype of Gc (vitamin D-binding protein): a cross-sectional study on 595 early postmenopausal women. Calcif Tissue Int 77: 15-22.

16. Lu L, Sheng H, Li H, et al, 2012 Associations between common variants in GC and DHCR7/NADSYN1 and vitamin D concentration in Chinese Hans. Hum Genet 131: 505-512. 